\title{
Obesity and overweight trends in Catalonia, Spain (1992-2003): gender and socio-economic determinants
}

\author{
Alicia García-Álvarez ${ }^{1}$, Lluís Serra-Majem ${ }^{1,2,3, *}$, Lourdes Ribas-Barba ${ }^{1}$, Conxa Castell ${ }^{3}$, \\ Marius Foz ${ }^{4}$, Ricardo Uauy ${ }^{5}$, Antoni Plasencia ${ }^{3}$ and Lluís Salleras ${ }^{6}$ \\ ${ }^{1}$ Community Nutrition Research Centre, University of Barcelona Science Park, Baldiri Reixac 4, Torre D 4A1, \\ 08028 Barcelona, Spain: ${ }^{2}$ Department of Clinical Sciences, University of Las Palmas de Gran Canaria, Las \\ Palmas de Gran Canaria, Spain: ${ }^{3}$ Division of Public Health, Department of Health, Generalitat of Catalonia, \\ Barcelona, Spain: ${ }^{4}$ Department of Internal Medicine, Autonomous University of Barcelona, Barcelona, Spain: \\ ${ }^{5}$ London School of Hygiene and Tropical Medicine, London, England: ${ }^{6}$ Department of Public Health, University \\ of Barcelona, Barcelona, Spain
}

Submitted 6 July 2007: Accepted 7 September 2007

\begin{abstract}
Objective: To evaluate the trends of overweight and obesity prevalences in the population of Catalonia, Spain, aged 18-75years, and the influence of socio-economic determinants on these prevalence trends.

Design: Analysis based on data from two representative population-based cross-sectional surveys.

Setting: Data from the two Evaluations of Nutritional Status in Catalonia (ENCAT 1992-93 and ENCAT 2002-03), Spain. Weights and heights were obtained by direct measurement in standardised conditions by trained interviewers. Overweight and obesity were defined using body mass index (BMI) and waist circumference (WC), categorised according to WHO criteria.

Subjects: In total, 1015 men and 1233 women from ENCAT 1992-93, and 791 men and 924 women from ENCAT 2002-03.

Results: Mean BMI and mean WC were higher in males in 2002-03 as compared to 1992-93, while for females mean BMI was lower except for the youngest group, and mean WC was higher. In men, overall BMI overweight prevalence remained stable (from $44.1 \%$ to $43.7 \%$ ), while obesity increased (from $9.9 \%$ to $16.6 \%$ ); total WC overweight remained stable (from 21.7 to $23.8 \%$ ), while WC obesity increased (from $13.1 \%$ to $24.4 \%$ ). In women, overall BMI overweight increased (from $29.1 \%$ to $30.1 \%$ ), whereas BMI obesity remained stable (from $15.0 \%$ to $15.2 \%$ ); total WC overweight decreased (from $21.8 \%$ to $17.7 \%$ ), while WC obesity increased (from $24.5 \%$ to $31.1 \%$ ). The socio-economic and education variables had an influence on BMI and WC overweight and obesity rates mainly on females in both surveys and on the youngest men only in the 1992-93 survey.

Conclusions: Ten-year trends indicate that Catalan males are getting bigger overall (BMI) and around the waistline (WC), while Catalan females only have bigger waistlines (WC). BMI male obesity prevalence has overtaken that of females. WC obesity continues to be more prevalent among females than males.
\end{abstract}

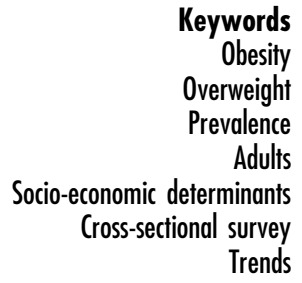

Overweight and obesity are recognised as public health problems worldwide and as major causes of preventable ill health ${ }^{1}$. Total obesity is the sixth most important risk factor contributing to the overall burden of disease worldwide, being a major risk factor for chronic noncommunicable diseases such as hypertension, coronary heart disease, type 2 diabetes, dyslipidaemia, as well as to some hormone-dependent cancers ${ }^{2}$. Abdominal obesity is a strong predictor of coronary heart disease and related risk factors ${ }^{3}$. Overweight and obesity also have an important health cost associated with them ${ }^{2}$.

The World Health Organisation (WHO) recently reported 1.1 billion overweight individuals and 300 million obese individuals ${ }^{1}, 10 \%$ of which are overweight or obese children ${ }^{4}$. Overweight and obesity prevalence rates are increasing in both developed and developing societies $^{1}$. The WHO reported that, since 1980, obesity prevalence rates have increased threefold in Northern 
America (in the United States over $65 \%$ of adults are now overweight or obese ${ }^{5}$ ), the United Kingdom, Central and Eastern Europe, Pacific Islands, Australia and China ${ }^{1}$.

In Spain, adult, adolescent and child obesity prevalence has also increased in the last decade ${ }^{6,7}$. The group of children aged between 6 and 13 years and the group of women aged over- 45 years are the groups with the highest risk of obesity; obesity prevalence is higher among males during years of growth and development ${ }^{6,7}$, while in the over 45-year group it is significantly higher in females ${ }^{6,8}$. In a recent study in Southern Spain, the authors found that a larger proportion of men were overweight compared to women, but the opposite was found for obesity 9 . In 2004, the results from the DORICA Study ${ }^{10}$ showed that the obesity prevalence of the NorthEastern region of Spain (which includes Catalonia) was $8.5 \%$ for men and $13.8 \%$ for women, which were the lowest out of the eight regions included in the study ${ }^{6}$.

Numerous studies have shown that obesity is more frequent in the less socially advantaged population groups, regardless of the variable used to classify socioeconomic status (SES); these differences in the prevalence of obesity by SES have been observed in both men and women, but are stronger and more consistent in women $^{11}$. The WHO's MONICA Study showed that the prevalence of obesity is higher among adults and children of low SES ${ }^{12}$. In Spain, in 1987, a group of researchers found a higher prevalence of obesity among the population of a lower educational level $^{8}$; in the period 1987-97, the same researchers found a higher obesity prevalence in individuals with elementary education, and that the obesity prevalence proportion associated with elementary education increased in women and decreased in men ${ }^{13}$. Moreover, the SEEDO'97 Study in Spain showed higher obesity rates in men and women with low educational level, and also that older women with low educational level and low income seemed to be the most susceptible group to weight gain ${ }^{2}$. Adding to this evidence, a significant inverse relationship between SES and overweight and obesity was found by the AVENA (Alimentación y Valoración del Estado Nutricional de los Adolescentes Españoles) Study ${ }^{14}$, although only in male adolescents.

Overweight and obesity also have a sociodemographic component. In this respect, the SEEDO'97 Study in Spain also showed differences in the distribution of the obesity prevalence by area of residence and geographical zones ${ }^{15}$.

Other well-known factors that influence the development of obesity are physical inactivity ${ }^{16,17}$, overconsumption of energy-dense diets (which has been shown to be associated with low SES $)^{18}$ and genetic factors (although some authors do not agree to this) ${ }^{5}$.

The objective of the present paper is to evaluate the trends (1992-2003) of overweight and obesity prevalences in the 18-75-year-old population of Catalonia,
Spain, and the influence of socio-economic and sociodemographic determinants on these prevalence trends.

\section{Material and Methods}

\section{Sample and subjects}

The data analysed in this paper belong to the 1992-93 and the 2002-03 cross-sectional Evaluations of the Nutritional Status of the Catalan Population (ENCAT 1992-93 and ENCAT 2002-03) $)^{15,19}$. ENCAT is a regional survey carried out periodically by the Department of Health of the Catalan Government and co-ordinated by the Centre for Research on Community Nutrition of the University of Barcelona. The theoretical random sample population and sample size have been described elsewhere $^{15,19}$, comprising the population source of residents in the official census. The samples were stratified according to household and randomised into subgroupings with municipalities being the primary sample units, and individuals within these municipalities comprising the final sample units. The valid response rate for the first survey was $69 \%$ and for the second $65 \%$.

Adults from each representative sample within the age of 18-75 years were included in the analysis of this study ( $n$ in ENCAT $1992-93=2248$ and $n$ in ENCAT $2002-03=1715)$.

\section{Data collection procedures and variables of the study}

In both surveys, dietitians were trained on standardisation of criteria and methodology before data collection, in order to reduce inter-observer measurement variability. The data were collected from 1992 to 1993 and from 2002 to 2003 through questionnaires and anthropometric measurements during a home interview.

In order to analyse the influence of the socio-economic determinants on the prevalence of overweight and obesity, the following variables were used and rearranged according to the following categories ${ }^{2}$ :

1. Socio-economic level (SEL) (occupation of the subject): (a) low: the non-classifiable, army, agricultural sector, service sector and non-qualified labourers; (b) medium: qualified labourers, foremen, rest of administrative, commercial and technical staff and medium-level technicians; (c) high: high-level technicians, directors/ managers, self-employed professionals, business owners or self-employed individuals without staff, business owners or self-employed individuals with staff.

2. Education level of the subject and of the family's head member (ELS and ELH): (a) low: primary school incomplete or illiterate ( $<6$ years at school); (b) medium: primary school completed, secondary school or further education (6-12 years of education); (c) bigh: high school, college or university degree $(>12$ years of education). 
The sociodemographic determinants included (1) gender, (2) age group (18-24, 25-44, 45-64 and 65-75 years) and (3) population of residence size $(<10,000$ inhabitants, 10,000-100,000 inhabitants and $>100,000$ inhabitants).

\section{Antbropometric measurements}

Body mass index

Weight and height were measured with a portable spring scale and a metric tape (Kawe ${ }^{\mathbb{C}}$ model). The individuals were measured in standardised conditions, wearing underwear and no shoes. Weight was measured in kilograms, scale measurement error $\pm 100 \mathrm{~g}$. Height was measured standing and head in the Frankfurt horizontal position, expressed in centimetres, instrumental measurement error $\pm 0.1 \mathrm{~cm}$. Body mass index (BMI) was calculated using weight and height and categorised according to WHO criteria $^{20}$ so that overweight was defined as BMI $\geq 25.0$ to BMI $<30.0 \mathrm{~kg} \mathrm{~m}^{-2}$ and obesity as BMI $\geq 30 \mathrm{~kg} \mathrm{~m}^{-2}$.

\section{Waist circumference}

Waist circumference (WC) was measured with a nonelastic metric tape halfway between the lower border of the ribs and the iliac crest on a horizontal plane. Measurements were recorded to the nearest $0.1 \mathrm{~cm}$ and categorised according to WHO criteria, so that men with a WC $94.0-101.9 \mathrm{~cm}$ and women with a WC $80.0-87.9 \mathrm{~cm}$ were classified as overweight, and men with a WC $\geq$ $102.0 \mathrm{~cm}$ and women with a WC $\geq 88.0 \mathrm{~cm}$ were classified as obese ${ }^{20}$.

\section{Statistical analysis}

All analyses were performed with SPSS 12.0. Proportions of overweight and obesity were estimated for each sample separately and stratified by gender and age (to control for its potential confounding effects). The age distribution of the whole Catalan population in 1992-93 was used as a reference. The proportions from the two surveys were compared using the $\chi^{2}$ statistic test and the means were compared using the $t$-test, considering $P$-values $<0.05$ for significance.

\section{Results}

The sample characteristics of the two surveys are presented in Table 1: the total number of subjects by gender, age group and each socio-economic/sociodemographic variable category.

Table 2 shows the mean, standard deviations and 5 th-95th percentiles of BMI and WC by gender and age group. In 2002-03, male mean BMI was higher than in 1992-93, although the observed difference was significant only for individuals aged 25-44 years (from 25.2 to 25.9) and 45-64 years (from 26.7 to 27.4), and male mean WC was significantly higher in all age groups; for females the observed decreasing trends in mean BMI in most age groups (except for the youngest) were not significant, while mean WC was significantly higher only in the youngest (from 70.3 to 72.7 ) and eldest (from 92.2 to 95.3) individuals. These results are shown in Figs 1 and 2, which also show how mean BMI and WC increase as age progresses in both genders. Percentiles 50, 75 and 95 of BMI showed increases in males from all age groups and decreases in females (except for the youngest group). As for WC, percentiles 50, 75 and 95 showed increases in males and females of all age groups.

Table 3 shows overall by-gender and by-survey BMI and WC overweight and obesity prevalences; it can be observed that the overall prevalence of BMI obesity increased significantly only in males (6.7 percentage points, from $9.9 \%$ to $16.6 \%$ ) in the 10 -year period, while that of WC obesity increased in both sexes (11.3 percentage points for males - from $13.1 \%$ to $24.4 \%$, and 6.6 for females - from $24.5 \%$ to $31.1 \%$ ). Table 3 also shows BMI and WC overweight and obesity prevalences when age, SEL, education level and population of residence size are considered.

When considering the variable 'age', Table 3 shows that in ENCAT 1992-93, the highest prevalence of BMI overweight was found in both males and females aged 45-64 years, which was also the case for female but not for male WC overweight; while in ENCAT 2002-03, only an increase in female BMI overweight and male WC overweight were observed with progressing age. Regarding obesity, both surveys showed an increase in the prevalence of BMI and WC obesity with progressing age in both sexes (note the high prevalence of WC obesity among the eldest men and women in 2002-03, 49.6\% and $70.9 \%$ respectively). The between-survey comparison showed significant changes only in male BMI overweight and male WC obesity rates, demonstrating alarming increases in the latter rates (i.e. from $1.3 \%$ to $6.0 \%$ in the 18-24-year-old group).

Regarding the variable 'socio-economic level' (SEL), the differences observed in BMI and WC overweight and obesity prevalences of the different SEL groups were significant only in females of both surveys, WC obesity being highest in the low SEL group (Table 3). In ENCAT 1992-93, SEL was inversely related to the prevalence of BMI obesity, but only significantly in females; this inverse relationship was not observed among SEL groups in ENCAT 2002-03. WC obesity was only inversely related with SEL in females of both surveys and the differences among SEL groups were significant (Table 3). The between-survey comparison showed significant increases in male BMI and WC obesity (from 8.3\% to 16.5\% and from $13.3 \%$ to $26.3 \%$ ) and female WC obesity (from $15.3 \%$ to $19.3 \%)$.

With regard to the variable 'education level of the subjects' (ELS), in ENCAT 2002-03, Table 3 shows an 
Table 1 Sample characteristics of the two ENCAT surveys

\begin{tabular}{|c|c|c|c|c|}
\hline \multirow[b]{2}{*}{ Variables } & \multicolumn{2}{|c|}{ ENCAT 1992-93 } & \multicolumn{2}{|c|}{ ENCAT 2002-03 } \\
\hline & $n$ & $\%$ & $n$ & $\%$ \\
\hline \multicolumn{5}{|l|}{ Gender } \\
\hline Males & 1015 & 45.2 & 791 & 46.1 \\
\hline Females & 1233 & 54.8 & 924 & 53.9 \\
\hline \multicolumn{5}{|l|}{ Age group (years) } \\
\hline $18-24$ & 526 & 23.4 & 276 & 16.1 \\
\hline $25-44$ & 801 & 35.6 & 654 & 38.1 \\
\hline $45-64$ & 668 & 29.7 & 557 & 32.5 \\
\hline $65-75$ & 253 & 11.3 & 228 & 13.3 \\
\hline \multicolumn{5}{|l|}{$\mathrm{BMI}^{*}\left(\mathrm{~kg} \mathrm{~m}^{-2}\right)$} \\
\hline$<18.5$ (underweight) & 37 & 1.6 & 33 & 1.9 \\
\hline 18.5 to $<25.0$ (normal) & 1119 & 49.8 & 787 & 45.9 \\
\hline 25.0 to $<30.0$ (overweight) & 807 & 35.9 & 624 & 36.4 \\
\hline$\geq 30.0$ (obese) & 285 & 12.7 & 271 & 15.8 \\
\hline \multicolumn{5}{|l|}{$\mathrm{WC}+(\mathrm{cm})$} \\
\hline Normal & 1312 & 59.4 & 878 & 52.2 \\
\hline Overweight & 484 & 21.5 & 349 & 20.0 \\
\hline Obese & 430 & 19.1 & 477 & 27.8 \\
\hline \multicolumn{5}{|c|}{ Socio-economic level (SEL - occupation) } \\
\hline Low & 666 & 30.5 & 258 & 15.2 \\
\hline Medium & 964 & 44.1 & 627 & 36.9 \\
\hline High & 557 & 25.5 & 812 & 47.8 \\
\hline \multicolumn{5}{|l|}{ Education level subject (ELS) } \\
\hline Low & 486 & 21.6 & 233 & 13.6 \\
\hline Medium & 1154 & 51.3 & 861 & 50.3 \\
\hline High & 608 & 27.0 & 618 & 36.1 \\
\hline \multicolumn{5}{|c|}{ Education level head of family (ELH) } \\
\hline Low & 654 & 29.1 & 264 & 15.5 \\
\hline Medium & 1145 & 50.9 & 925 & 54.3 \\
\hline High & 449 & 20.0 & 513 & 30.1 \\
\hline \multicolumn{5}{|l|}{ Population size (inhabitants) } \\
\hline$<10,000$ & 294 & 13.1 & 379 & 22.1 \\
\hline $10,000-100,000$ & 604 & 26.9 & 541 & 31.5 \\
\hline$>100,000$ & 1350 & 60.1 & 795 & 46.4 \\
\hline Total $(n)$ & 2248 & 100.0 & 1715 & 100.0 \\
\hline
\end{tabular}

*Body mass index - according to WHO classification (1998).

tWaist circumference - according to WHO classification (1998): normal: $<94 \mathrm{~cm}$ for males and $<80 \mathrm{~cm}$ for females; overweight: $\geq 94$ to $<102 \mathrm{~cm}$ for males and $\geq 80$ to $<88 \mathrm{~cm}$ for females; obese: $\geq 102 \mathrm{~cm}$ for males and $\geq 88 \mathrm{~cm}$ for females.

ENCAT - Evaluation of Nutritional Status in Catalonia.

inverse relationship with BMI overweight and obesity prevalence, but with differences among ELS groups only significant in females (note a female BMI obesity prevalence of $36.6 \%$ in the lowest ELS group); this inverse relationship was observed between ELS and male and female WC obesity but not WC overweight (note the high male and female WC obesity rates in the lowest ELS group, $34.3 \%$ and $69.5 \%$ respectively). The betweensurvey comparison revealed significant differences in both BMI and WC overweight and obesity for both genders. It is worth noticing that while male and female WC overweight seem to have increased among the highest ELS group (from $15.5 \%$ to $23.9 \%$ and from $11.8 \%$ to $14.9 \%$ respectively), female WC obesity prevalence in the lowest ELS group increased by 20 percentage points (from $49.3 \%$ to $69.5 \%$ ).

The variable 'education level of the family head member' (ELH) showed in ENCAT 2002-03 a significant inverse relationship with BMI overweight, BMI obesity and WC obesity in females (Table 3). Females whose family head member had a medium education level presented the highest WC overweight prevalence compared to females whose family head member had a low or high ELH (23.9\% in 1992-93 and 19.0\% in 2002-03). The between-survey comparison revealed significant differences in both BMI and WC overweight and obesity for both genders. Male and female BMI overweight, male and female BMI obesity, male WC overweight and male and female WC obesity rates increased in the low and high ELH groups, while in the medium ELH group the increase was only observed in male BMI overweight (from $9.6 \%$ to $17.5 \%$ ) and male and female WC obesity (from $12.9 \%$ to $24.9 \%$ and from $23.3 \%$ to $30.2 \%$ respectively). The difference of 27 percentage points in female WC obesity prevalence in the low ELH group is worth noticing (from 35.1 in ENCAT 1992-93 to 61.9\% in ENCAT 2002-03).

Regarding 'population of residence size', only ENCAT 2002-03 differences observed in female WC overweight and obesity were significant ('within-survey comparison', 
Table 2 Mean, standard deviation and percentiles of body mass index and waist circumference, by gender, age and survey (ENCAT 1992-93 and ENCAT 2002-03)

\begin{tabular}{|c|c|c|c|c|c|c|c|c|c|c|c|c|c|c|c|c|c|c|}
\hline \multirow[b]{2}{*}{ Gender } & \multirow[b]{2}{*}{ Age (years) } & \multicolumn{2}{|r|}{$n$} & \multicolumn{2}{|c|}{ Mean } & \multirow[b]{2}{*}{$t$-Test } & \multicolumn{2}{|c|}{ SD } & \multicolumn{2}{|c|}{ P5 } & \multicolumn{2}{|c|}{ P25 } & \multicolumn{2}{|c|}{ P50 } & \multicolumn{2}{|c|}{ P75 } & \multicolumn{2}{|c|}{ P95 } \\
\hline & & 1992-93 & $32002-03$ & $1992-93$ & $2002-03$ & & 1992-93 & $2002-03$ & 1992-93 & $2002-03$ & 1992-93 & $2002-03$ & 1992-03 & $2002-03$ & 1992-93 & 2002-03 & 1992-93 & 2002-03 \\
\hline \multicolumn{19}{|c|}{ Body mass index $\left(\mathrm{kg} \mathrm{m}^{-2}\right)$} \\
\hline \multirow[t]{4}{*}{ Males } & $18-24$ & 237 & 116 & 23.4 & 24.0 & $\mathrm{~ns}^{*}$ & 2.8 & 3.8 & 19.2 & 19.6 & 21.4 & 21.4 & 22.9 & 23.2 & 25.2 & 25.7 & 28.7 & 32.5 \\
\hline & $25-44$ & 359 & 309 & 25.2 & 25.9 & $S^{\star *}$ & 3.1 & 3.7 & 20.4 & 20.3 & 23.1 & 23.2 & 25.0 & 25.4 & 27.1 & 28.1 & 31.1 & 33.0 \\
\hline & $45-64$ & 312 & 250 & 26.7 & 27.4 & $s$ & 3.4 & 3.4 & 21.4 & 22.0 & 24.6 & 25.5 & 26.5 & 27.3 & 28.6 & 29.1 & 31.9 & 33.2 \\
\hline & $65-75$ & 107 & 116 & 26.9 & 27.7 & ns & 3.5 & 3.6 & 21.7 & 22.6 & 24.8 & 24.8 & 26.2 & 27.6 & 29.8 & 30.4 & 33.7 & 33.6 \\
\hline \multirow{4}{*}{ Females } & $18-24$ & 289 & 160 & 22.0 & 22.1 & ns & 2.6 & 3.1 & 18.5 & 18.2 & 20.1 & 19.9 & 21.5 & 21.5 & 23.6 & 24.3 & 26.6 & 27.8 \\
\hline & $25-44$ & 442 & 345 & 24.2 & 23.9 & ns & 4.1 & 4.1 & 19.3 & 18.9 & 21.5 & 20.9 & 23.5 & 23.0 & 26.1 & 26.1 & 31.2 & 31.2 \\
\hline & $45-64$ & 356 & 307 & 27.7 & 26.9 & ns & 4.5 & 4.7 & 21.2 & 19.9 & 24.7 & 23.6 & 27.2 & 26.3 & 30.2 & 29.8 & 36.0 & 35.3 \\
\hline & $\begin{array}{l}65-75 \\
\text { mference }(\mathrm{cm})\end{array}$ & 146 & 112 & 28.9 & 28.3 & ns & 6.2 & 4.4 & 20.5 & 19.3 & 25.2 & 25.8 & 28.7 & 28.6 & 31.4 & 30.5 & 38.7 & 36.3 \\
\hline \multicolumn{19}{|c|}{ Waist circumference (cm) } \\
\hline \multirow{3}{*}{ Males } & $\begin{array}{l}18-24 \\
25-44\end{array}$ & 359 & 309 & $\begin{array}{l}81.8 \\
88.4\end{array}$ & $\begin{array}{l}84.6 \\
91.5\end{array}$ & $\mathrm{~s}$ & $\begin{array}{l}0.0 \\
9.1\end{array}$ & 11.1 & 74.9 & 74.0 & 82.0 & $\begin{array}{l}10.9 \\
83.9\end{array}$ & $\begin{array}{l}81.0 \\
88.3\end{array}$ & $\begin{array}{l}00.0 \\
91.0\end{array}$ & 94.4 & $\begin{array}{l}90.5 \\
97.6\end{array}$ & $\begin{array}{r}91.1 \\
104.1\end{array}$ & 110.7 \\
\hline & $45-64$ & 312 & 250 & 95.2 & 97.7 & $s$ & 10.5 & 10.9 & 79.6 & 81.3 & 88.3 & 92.0 & 95.0 & 97.1 & 102.0 & 104.3 & 111.0 & 117.5 \\
\hline & $65-75$ & 107 & 116 & 97.0 & 102.8 & $\mathrm{~s}$ & 9.3 & 10.8 & 78.7 & 83.6 & 91.6 & 96.0 & 97.5 & 102.0 & 103.2 & 111.0 & 111.6 & 121.4 \\
\hline \multirow[t]{4}{*}{ Females } & $18-24$ & 289 & 160 & 70.3 & 72.7 & $\mathrm{~s}$ & 7.0 & 7.9 & 60.6 & 62.0 & 66.0 & 67.0 & 69.0 & 71.0 & 74.0 & 77.2 & 84.0 & 87.0 \\
\hline & $25-44$ & 442 & 345 & 77.4 & 78.8 & ns & 9.9 & 11.3 & 64.0 & 65.0 & 71.0 & 70.8 & 75.5 & 76.0 & 83.0 & 85.0 & 95.6 & 100.0 \\
\hline & $45-64$ & 356 & 307 & 87.1 & 86.9 & ns & 10.9 & 12.8 & 69.1 & 68.1 & 80.0 & 77.0 & 85.5 & 87.0 & 94.0 & 95.5 & 105.9 & 110.5 \\
\hline & $65-75$ & 146 & 112 & 92.2 & 95.3 & $s$ & 10.0 & 11.6 & 76.5 & 74.0 & 84.5 & 88.0 & 91.8 & 95.0 & 99.2 & 104.9 & 111.5 & 114.0 \\
\hline
\end{tabular}

${ }^{*}$ ns - non-significant difference; ${ }^{* *} \mathrm{~s}-$ significant difference $(P$-value $<0.05)$.

$t t-$ Test used for between-survey comparison of BMI and WC means.

= standard deviation; $P$ = percentile; ENCAT - Evaluation of Nutritional Status in Catalonia 


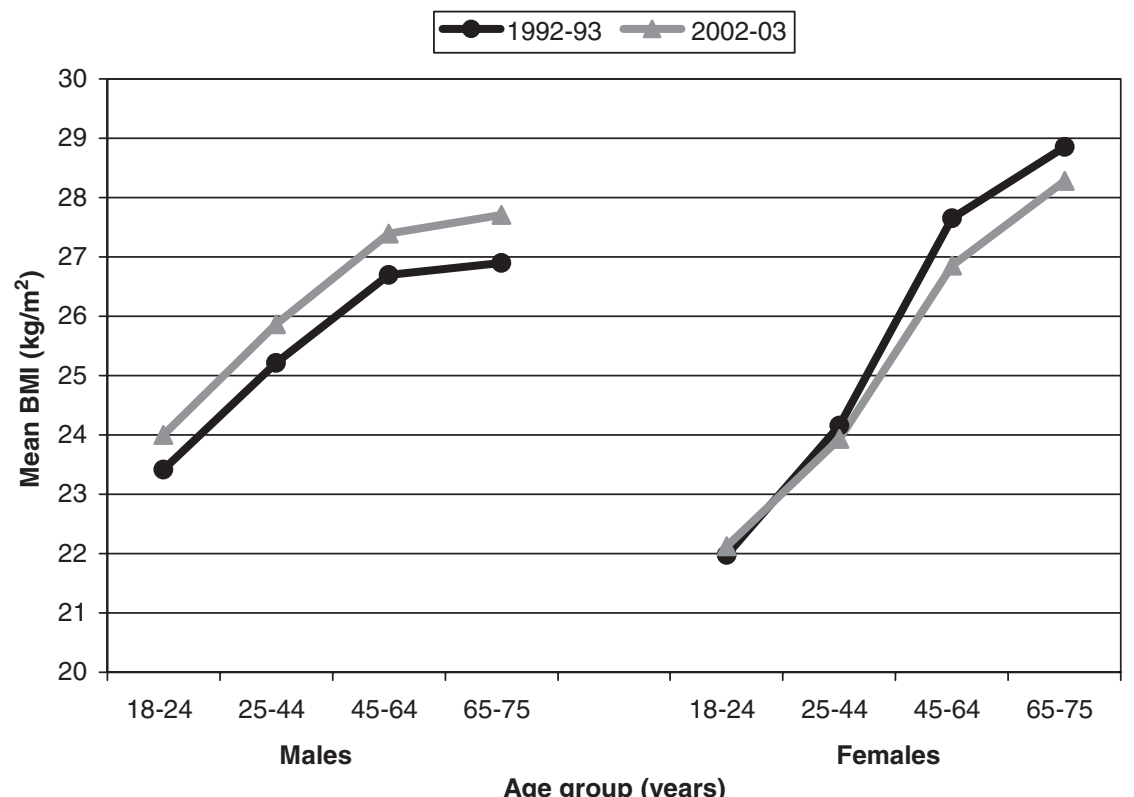

Fig. 1 Mean BMI by gender, age and survey year (ENCAT 1992-93 and 2002-03) (BMI - body mass index; ENCAT - Evaluation of Nutritional Status in Catalonia)

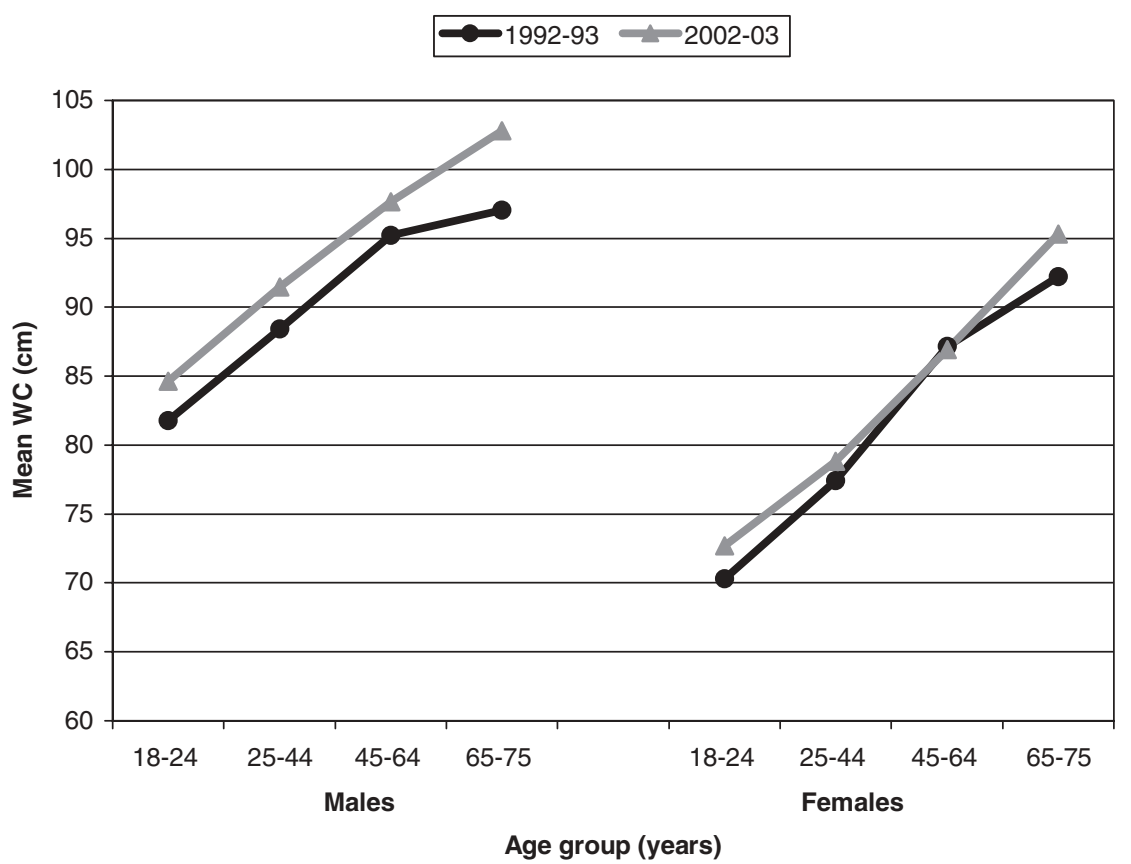

Fig. 2 Mean WC by gender, age and survey year (ENCAT 1992-93 and 2002-03) (WC - waist circumference; ENCAT Evaluation of Nutritional Status in Catalonia)

Table 3). The between-survey comparison showed significant differences in all prevalences except for males BMI and WC obesity; it is worth mentioning that, in the $<10,000$ inhabitants group, while female BMI and WC overweight rates decreased (from 32.5 to $27.8 \%$ and from 22.5 to $12.9 \%$, respectively), female $\mathrm{BMI}$ and $\mathrm{WC}$ obesity rates increased (from 15.6 to 18.0 and from 31.1 to $38.6 \%$, respectively).

Table 4 shows how the ENCAT 2002-03 BMI and WC overweight and obesity prevalences change when adjusting by the ENCAT 1992-93 SEL, ELS, ELH and population of residence size distributions. It is apparent 
Table 3 Overweight and obesity prevalence (BMI and WC) by gender, survey year (ENCAT 1992-93 and ENCAT 2002-03), socio-economic and sociodemographic characteristics

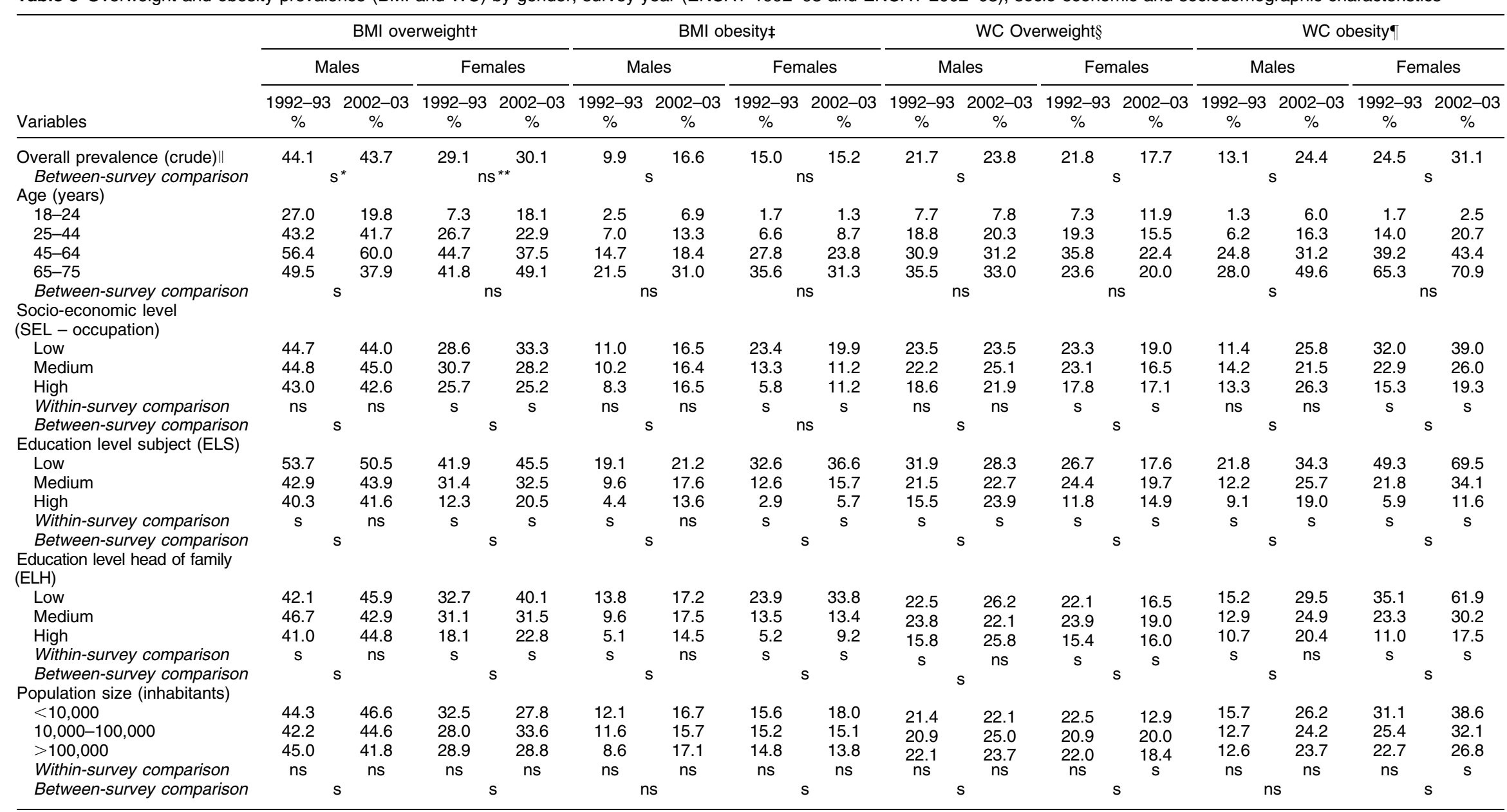

"s: $\chi^{2}$ test - significant difference $(P \leq 0.05)$; ${ }^{*} n s: \chi^{2}$ test - non-significant difference tBMl 25.0 to $\leq 30.0 \mathrm{~kg} \mathrm{~m}^{-2}$;

$\neq B M I \geq 30.0 \mathrm{~kg} \mathrm{~m}^{-2}$

\$WC 94 to $<102 \mathrm{~cm}$ for males and 80 to $<88 \mathrm{~cm}$ for females.

IWC $\geq 102 \mathrm{~cm}$ for males and $\geq 88 \mathrm{~cm}$ for females.

ISee Table 4 for adjusted prevalences.

ENCAT - Evaluation of Nutritional Status in Catalonia; BMI - body mass index; WC - waist circumference. 
that male and female BMI obesity increased to its highest when standardised by the ENCAT 1992-93 ELS and population size (from $16.6 \%$ to $17.8 \%$ and from $15.2 \%$ to $19.6 \%$, respectively), while male and female WC obesity increased to its highest when standardised by the ENCAT 1992-93 ELS (from 24.4\% to $26.7 \%$ and from $31.1 \%$ to $39.2 \%$, respectively). Male and female BMI and WC obesity decreased when adjusted by the ENCAT 1992-93 SEL distribution.

\section{Discussion}

The WHO recognises that the main limiting factors when comparing epidemiological studies on the prevalence of overweight and obesity are the following: the different criteria to define the cut-offs, the variation in age groups considered, the time interval for collection of data and study comparisons based on reported weight and height ${ }^{20}$. This study is based on the 1992-93 and 2002-03 ENCAT surveys, which were carried out on representative random samples of the Catalan population. Both surveys used the same anthropometric measurement procedures (weight, height and WC were measured instead of reported) and socio-economic factors, and allow for comparison of the same age groups (18-75 years).

The WHO has recommended BMI as a good index of total overweight and obesity ${ }^{20}$, although it gives no information about body fat distribution, while WC reflects abdominal visceral fat distribution. Nevertheless, the two measures are highly correlated ${ }^{21}$. It has been shown that changes in WC accompany changes in cardiovascular risk factors especially in the elderly ${ }^{22}$. Research has also shown that WC can also predict morbidity and mortality, considering it a better measure of obesity than BMI, since it is a simple and easy measurement ${ }^{23}$; WC is even more strongly associated with metabolic abnormalities and healthcare costs than $\mathrm{BMI}^{21}$. A single WC measurement has been suggested to be used to identify individuals who should seek and be offered weight management ${ }^{24}$. We have used both BMI and WC measures to define total and central overweight and obesity in order to have a more complete overall picture of the problem in the Catalan population.

This study has shown that in Catalonia, in 2002-03, mean BMI in males was higher than in 1992-93, and that of females was lower (except for the youngest group); on the other hand, overall prevalence of BMI overweight and obesity were $43.7 \%$ and $16.6 \%$; respectively in males and $30.1 \%$ and $15.2 \%$ respectively in females. When comparing these figures with those of the 2002 IOTF report for Spain (1998-2000), we observe that overweight was lower in Catalonia in both genders (in Spain $48 \%$ for males and $40 \%$ for females), while obesity was higher for Catalan males and females (in Spain $12 \%$ and $15 \%$, respectively $)^{25}$. Therefore, in terms of gender, this study 
shows that overweight and obesity are more prevalent in men (obesity was more prevalent in women 13 years ago, but male obesity has caught up and overcome the female prevalence). These findings are in agreement with other literature available from developed countries, which suggests that women hold a more negative attitude towards obesity than men and they are also more heavily influenced by the public negative view towards obesity, thus spending more time, effort and money on the ideal thinner shape ${ }^{26}$.

Regarding WC overweight and obesity prevalences, this study has shown that in ENCAT 2002-03, mean WC was higher in males and females as compared to ENCAT 1992-93 (except for the female group aged 45-64 years). In men, overall WC overweight increased (from $21.7 \%$ in 1992-93 to $23.8 \%$ in 2002-03) as well as overall WC obesity (from $13.1 \%$ to $24.4 \%$ ) increased. In women, overall WC overweight decreased (from $21.8 \%$ to $17.7 \%$ ), while overall WC obesity increased (from 24.5\% to $31.1 \%$ ). In other words, our results on BMI and WC overweight and obesity suggest that Catalan men are getting bigger overall and also specifically around the waist, while Catalan women are getting thinner overall but with bigger waistlines. They also show that WC obesity is increasing more rapidly than BMI obesity and, while BMI obesity is more prevalent among men, WC obesity is more prevalent among women. These findings agree with those of several recent studies carried out in Northern Europe ${ }^{21,27}$.

There are few studies that examine the possible relationship of SES and overweight and obesity prevalence, and even fewer for the actual distribution of its prevalence into the SES groups ${ }^{21,26,28}$. Although comparisons are not directly possible, there are three studies that show that obesity rates have been increasing for decades and are in line with our findings in that the prevalence of obesity is higher for the lower SES groups (two of these studies use education ${ }^{29,30}$ and one uses income ${ }^{28}$ ) and for men. Data from the ENCAT 2002-03 survey showed an increasing trend in the prevalence of BMI obesity in all male SEL (using occupation) groups as compared to ENCAT 1992-93, whereas female BMI obesity prevalence only increased in the high SEL group (although not significantly). The analysis showed that SEL had no influence on male BMI overweight or obesity prevalence, and that it only had an influence on BMI overweight and obesity prevalence among the oldest females (45-64 years and 65-75-year-olds), showing an inverse relationship (this further stratification by age group is not shown in the results). Referring to WC, in the 10-year period, only female WC overweight and obesity changed due to SEL, overweight decreased (being highest in the lowest SEL but no inverse relationship was observed) and obesity increased (highest in the lowest SEL, showing an inverse relationship). These findings are in agreement with numerous studies carried out in developed countries by which, overall, the prevalence of obesity is higher in lower SEL groups ${ }^{2,21,31}$. In developing countries the problem has been shown to be more prevalent among the highest SEL groups, some showing the inverse relationship between overweight/obesity and household amenities in both genders and occupational level in men $^{32}$.

Studies within the Spanish population have shown that the prevalence of obesity is higher among women and increases with age, particularly in the least educated female subgroups ${ }^{2,8}$, results that agree with the findings of the present study. The further stratification of each education level by age group (not shown in the results) revealed different prevalences from the overall male and female BMI obesity prevalences probably because the least educated people were mostly the older age groups with a higher obesity prevalence, which agrees with findings from the SEEDO'97 Study ${ }^{2}$. Regarding WC, we have shown that overweight basically increased in the male and female highest ELS groups, while obesity increased in all ELS groups, being highest in the lowest ELS one (inverse relationship) and most prevalent among the females of this group (reaching an alarming prevalence of $69.5 \%$ ), and affecting more prominently the 45-64-year-olds (again probably because the least educated people were mostly the older age groups with a higher WC obesity prevalence - analysis not shown). These results coincide with those obtained in recent studies $^{12,27,33}$, in particular, a study carried out in Spain, which showed an even higher prevalence of WC obesity in non-educated elderly females $(80.9 \%)^{33}$.

With regard to the influence of the area of residence (population size) on excess body weight, in the 10-year period, significant differences were found for BMI overweight in both sexes and for female BMI obesity; the differences were also significant for WC male and female overweight and female WC obesity. Females living in the smallest communities showed a decrease in BMI and WC overweight. However, this decrease was probably at the expense of an increase in female WC obesity. Studies carried out on the Spanish population ${ }^{2,34}$ disagree with our BMI findings by not showing significant differences on overweight and obesity when stratifying by population size, but no comparable results are available for WC prevalences.

Finally, SES has been found to be associated with dietary patterns and physical activity ${ }^{26,31,35,36}$. For example, showing more disadvantaged population groups generally have a poorer-quality diet (e.g. higher fat intake and lower vegetable consumption) than higher SES groups, which may partly explain the inverse association between SES and obesity demonstrated in some studies ${ }^{26,35}$. Other studies have evaluated how money expenditure on food can assist in the achievement of a healthy $\operatorname{diet}^{26,37}$. The inverse relationship between energy density and energy cost suggests that 'obesity-promoting' foods are simply 
those that offer the most dietary energy at the lowest cost. The relative cost has also been taken into account in the literature which increases even further the cost of the healthy diet for the low-income families ${ }^{26}$. The present study has not considered diet, physical activity, income (at least not directly), expenditure on food or food costs in its analysis (which was merely descriptive and far from suggesting causality due to the cross-sectional nature of the data); therefore, the authors recognise the need for a further and more robust analysis that involves all these lifestyle variables known to affect the relationship between prevalence of excess body weight and SES. In addition, self-reported occupation and education level may be over or under estimated. However, this probably has not significantly modified the classification of the participants into the three SES groups. Moreover, this study has not adjusted WC for BMI, which should be done due to the influence a high BMI can have on a high $\mathrm{WC}^{21}$. In spite of the mentioned limitations, we believe that our findings contribute to the evidence needed to guide public health policy makers in the design and implementation of preventive campaigns against the increasing trends of overweight and obesity, paying special attention to males and low SEL and educationlevel groups, and small population of residence size (for male overweight and female obesity).

\section{Acknowledgements}

Sources of funding: This work was made possible by financing from the General Division of Public Health of the Generalitat of Catalonia's Department of Health, through a research agreement with the Fundación para la Investigación Nutricional (Nutrition Research Foundation).

Conflict of interest declaration: None of the authors had any conflicts of interest in connection with this study.

Authorship responsibilites: AGA was responsible for the statistical analysis, data interpretation and writing of the paper; LSM was director of the study and revised the paper providing expert advice on data interpretation and discussion of the paper; LRB was coordinator of the study and responsible of the databases and revised the paper providing expert advice on data interpretation; CC participated in the study concept and design; MF, RU, $\mathrm{AP}$ and LS revised the paper providing expert advice in its discussion.

Guarantor: Lluís Serra-Majem.

Acknowledgements: Part of this analysis belongs to Alicia García-Álvarez's MSc thesis at the London School of Hygiene and Tropical Medicine (2004-05) supervised by Ricardo Uauy.

Special acknowledgement is made to all those persons who were interviewed, and whose collaboration made the realisation of these surveys possible.
Research Group on the Evaluation and Monitoring of the Nutritional Status in the Catalan Population: Lluís Serra-Majem, Director (University of Las Palmas de Gran Canaria); Lourdes Ribas-Barba, Coordinator (FIN-Nutrition Research Foundation, Barcelona Science Park); Gemma Salvador (Generalitat of Catalonia); Conxa Castell (Generalitat of Catalonia); Blanca Román- Viñas (FIN, Barcelona Science Park); Jaume Serra (Generalitat of Catalonia); Lluís Jover (University of Barcelona); Ricard Tresserras (Generalitat of Catalonia); Blanca Raidó (FIN, Barcelona Science Park); Andreu Farran (CESNID, University of Barcelona); Joy Ngo (FIN, Barcelona Science Park); Mari Cruz Pastor (Hospital Germans Trias i Pujol, Badalona); Lluís Salleras (University of Barcelona); Ricardo Uauy (London School of Hygiene and Tropical Medicine (England), and Carmen Cabezas, Josep Lluís Taberner, Salvi Juncà, Josep Maria Aragay, Gonçal Lloveras Vallès († 2003), Antoni Plasencia (Generalitat of Catalonia).

\section{References}

1 World Health Organisation. Global Strategy on Diet, Physical Activity and Health, 2003. Available at http:// www.who.int/hpr/global.strategy.shtml (accessed 23 January 2007).

2 Aranceta J, Pérez Rodrigo C, Serra Majem LI, Ribas L, QuilesIzquierdo J, Vioque J, et al and Spanish Collaborative Group for the Study of Obesity. Influence of sociodemographic factors in the prevalence of obesity in Spain. The SEEDO'97 Study. European Journal of Clinical Nutrition 2001; 55 : $430-5$.

3 Rexrode KM, Carey VJ, Hennekens CH, Walters EE, Colditz GA, Stampfer MJ, et al. Abdominal adiposity and coronary heart disease in women. Journal of the American Medical Association 1998; 280: 1843-8.

4 Haslam DW, James WPT. Seminar: obesity. The Lancet 2005; 366: 1197-209.

5 Townsend MS. Obesity in low-income communities: prevalence, effects, a place to begin. Journal of the American Dietetic Association 2006; 106: 34-7.

6 Aranceta-Bartrina J, Serra-Majem LL, Foz-Sala M, MorenoEsteban B, and Grupo Colaborativo SEEDO. Prevalencia de obesidad en España. Medicina Clinica (Barcelona) 2005; 125: 460-6.

7 Serra Majem L, Ribas Barba L, Aranceta Bartrina J, Perez Rodrigo C, Saavedra Santana P, Pena Quintana L. Childhood and adolescent obesity in Spain. Results of the enKid study (1998-2000). Medicina Clínica (Barcelona) 2003; 121: $725-32$.

8 Gutiérrez-Fisac JL, Regidor E, Rodríguez C. Prevalencia de la obesidad en España. Medicina Clínica (Barcelona) 1994; 102: 10-13.

9 Mataix J, Lopez-Frias M, Martinez-de-Victoria E, LopezJurado M, Aranda P, Llopis J. Factors associated with obesity in an adult Mediterranean population: influence on plasma lipid profile. Journal of the American College of Nutrition 2005; 24: 456-65.

10 Aranceta J, Pérez Rodrigo C, Serra Majem L, Vioque J, Tur Marí JA, Mataix Verdú J, et al. Estudio DORICA: dislipemia, obesidad y riesgo cardiovascular. In: Aranceta J, Foz M, Gil B, Jover E, Mantilla T, Millán J, Monereo S, 
Moreno B, eds. Obesidad y riesgo cardiovascular. Estudio DORICA. Madrid: Panamericana, 2004; 125-56.

11 Sobal J, Stunkard AJ. Socioeconomic status and obesity: a review of the literature. Psychological Bulletin 1989; 105: 260-75.

12 Molarius A, Seidell JC, Sans S, Tuomilehto J, Kuulasmaa K. Educational level, relative body weight, and changes in their association over 10 years: an international perspective from the WHO, MONICA project. American Journal of Public Health 2000; 90: 1260-8.

13 Gutiérrez-Fisac JL, Regidor E, Banegas Banegas JR, Rodríguez Artalejo F. The size of obesity differences associated with education level in Spain, 1987 and 1995/97. Journal Epidemiology and Community Health 2002; 56: 457-60.

14 Moreno LA, Mesana MI, Fleta J, Ruiz JR, Gonzalez-Gross M, Sarria A, et al. Overweight, obesity and body fat composition in Spanish adolescents. The AVENA Study. Annals of Nutrition and Metabolism 2005; 49(2): 71-6.

15 Serra Majem L, Ribas Barba L, García Closas R, Ramon JM, Salvador G, Farran A, et al. Llibre Blanc: Avaluació de l'estat nutricional de la població catalana (1992-93). Barcelona: Departament de Sanitat i Seguretat Social, Generalitat de Catalunya, 1996.

16 Jakicic MJ, Otto AD. Physical activity considerations for the treatment and prevention of obesity. American Journal of Clinical Nutrition 2005; 82(Suppl. 1): S226-9.

17 Gutierrez-Fisac JL, Regidor E, Lopez Garcia E, Banegas Banegas JR, Rodriguez Artalejo F. The obesity epidemic and related factors: the case of Spain. Cadernos de Saude Publica 2003; 19(Suppl. 1): S101-10.

18 Rolls BJ, Drewnowski A, Ledikwe JH. Changing the energy density of the diet as a strategy for weight management. Journal of the American Dietetic Association 2005; 105(Suppl. 1): S98-103.

19 Serra Majem L, Ribas Barba L, Salvador Castell G, Castells Abat C, Román Viñas B, Serra J, et al. Avaluació de l'estat nutricional de la població catalana 2002-2003. Evolució dels hàbits alimentaris $i$ del consum d'aliments $i$ nutrients a Catalunya (1992-2003). Barcelona: Departament de Salut, Generalitat de Catalunya, 2006.

20 World Health Organization. Programme of Nutrition, Family and Reproductive Health. Obesity. Preventing and Managing the Global Epidemic. Report of a WHO consultation on obesity. Geneva: WHO, 1998.

21 Sarlio-Lähteenkorva S, Silventoinen K, Lahti-Koski M, Laatikainen T, Jousilahti P. Socio-economic status and abdominal obesity among Finnish adults from 1992 to 2002. International Journal of Obesity 2006; 30(11): $1653-60$

22 Turcato E, Bosello O, Di Francesco V, Harris TB, Zoico E, Bissoli L, et al., Institute et al Waist circumference and abdominal sagittal diameter as surrogates of body fat distribution in the elderly: their relation with cardiovascular risk factors. International Journal of Obesity Related Metabolic Disorders 2000; 24: 1005-10.
23 Lean MEJ, Han TS, Seidell JC. Impairment of health and quality of life in people with large waist circumference. The Lancet 1998; 351: 853-6.

24 Lean MEJ, Han TS, Morrison CE. Waist circumference as a measure for indicating need for weight management. British Medical Journal 1995; 311: 158-61.

25 IOTF, EASO. Obesity in Europethe case for action. London: International Obesity Task Force and European Association for the Study of Obesity, 2002.

26 Manios Y, Panagiotakos DB, Pitsavos C, Polychronopoulos $\mathrm{E}$, Stefanadis C. Implication of socio-economic status on the prevalence of overweight and obesity in Greek adults: the ATTICA study. Health Policy 2005; 74: 224-32.

27 Chen R, Tunstall-Pedoe $H$. Socioeconomic deprivation and waist circumference in men and women: the Scottish MONICA surveys 1989-1995. European Journal of Epidemiology 2005; 20: 141-7.

28 Choiniere R, Lafontaine P, Edwards AC. Distribution of cardiovascular disease risk factor by socioeconomic status among Canadian adults. Journal of Ayub Medical College 2000; 162: S13-24.

29 Berkman LF, Breslow L. Health and ways of living: the Alameda County Study. New York: Oxford University Press, 1983.

30 Lynch JW, Kaplan GA, Salonen JT. Why do poor people behave poorly? Variations in adults behaviours and psychosocial characteristics, by stage of socioeconomic life-course. Social Science and Medicine 1997; 44: 809-20.

31 Stam-Moraga M, Kolanowski J, Dramaix J, De Backer G, Kornitzer MD. Sociodemographic and nutritional determinants of obesity in Belgium. International Journal of Obesity Related Metabolic Disorders 1999; 23(Suppl. 1): S1-9.

32 Fezeu L, Minkoulou E, Balkau B, Kengne AP, Awah P, Unwin N, et al. Association between socioeconomic status and adiposity in urban Cameroon. International Journal of Epidemiology 2006; 35: 112-13.

33 Gutiérrez-Fisac JL, López E, Banegas JR, Graciani A, Rodríguez-Artalejo F. Prevalence of overweight and obesity in elderly people in Spain. Obesity Research 2004; 12: 710-15.

34 Aranceta J, Pérez Rodrigo C, Serra Majem LL, Ribas L, Quiles Izquierdo J, Vioque J, et al. Prevalencia de la obesidad en España: estudio SEEDO'97. Medicina Clínica (Barcelona) 1998; 111: 441-5.

35 Proper KI, Cerin E, Brown WJ, Owen N. Sitting time and socio-economic differences in overweight and obesity. International Journal of Obesity 2007; 31(1): 169-76.

36 Aranceta J, Pérez C, Marzana I, Egileor I, González de Galdeano L, Sáenz de Buruaga J. Food consumption patterns in the adult population of the Basque Country (EINUT-I). Public Health Nutrition 1998; 3: 185-92.

37 Drewnowski A, Specter SE. Poverty and obesity: the role of energy density and energy costs. American Journal of Clinical Nutrition 2004; 79: 6-16. 